\title{
Endovascular transvenous cure for ruptured brain arteriovenous malformations in complex cases with high Spetzler-Martin grades
}

\author{
Christina losif, MD, MSc, PhD, ${ }^{1}$ Georges A. C. Mendes, MD, ${ }^{1}$ Suzana Saleme, MD, ${ }^{1}$ \\ Sanita Ponomarjova, MD, ${ }^{1}$ Eduardo Pedrolo Silveira, MD, ${ }^{1}$ Francois Caire, MD, PhD, ${ }^{2}$ \\ and Charbel Mounayer, MD, PhD'
}

Departments of ${ }^{1}$ Interventional Neuroradiology and ${ }^{2}$ Neurosurgery, Dupuytren University Hospital, Limoges, France

\begin{abstract}
OBJECT Ruptured cerebral arteriovenous malformations (AVMs) with deep localization and high Spetzler-Martin grades are associated with considerable challenges regarding nidus eradication treatment. The authors report their experience with curative endovascular transvenous embolization in a series of patients harboring "untreatable" lesions.

METHODS Between January 2008 and June 2013, a transvenous endovascular embolization protocol was implemented at the authors' institution for consecutive patients with ruptured brain AVMs that were considered incurable by classic endovascular and surgical techniques. Therapeutic decision making was based on Spetzler-Martin grades, AVM location, type of venous drainage, and angioarchitectural evaluation. Complete exclusion of the nidus was the objective of treatment.

RESULTS Twenty patients (10 male and 10 female, mean age $36.7 \pm 17.7$ years) were included. Initial Spetzler-Martin grades were III-V for $90.0 \%$ of the patients. The lesions were deeply seated in $80 \%$ and in eloquent locations in $90 \%$ of cases. The preprocedural modified Rankin Scale score was $0-2$ for 12 of the 20 patients $(60.0 \%), 3$ for 2 patients $(10.0 \%)$, and 4 for 6 patients $(30.0 \%)$. The postprocedural clinical status was unchanged for all patients. The procedure was technically feasible in all cases. Procedure-related mortality was $0 \%$. Ninety percent of the patients were independent in their everyday lives (modified Rankin Scale Scores 0-2) at the 6-month follow-up. In all cases but one (95\%) the embolization was curative, confirmed by selective DSA at 6 months and 18 months postintervention.
\end{abstract}

CONCLUSIONS Single-session endovascular transvenous embolization seems to be a safe and effective curative treatment for patients harboring complex brain AVMs with high Spetzler-Martin grade.

http://thejns.org/doi/abs/10.3171/2014.9.JNS141714

KEY WORDS cerebral arteriovenous malformation; brain arteriovenous malformation; therapeutic embolization; intraventricular hemorrhage; intracranial hematoma; transvenous embolization; vascular disorders

$\mathrm{R}$ ECENT data from a prospective controlled trial have raised controversy regarding the benefit of invasive treatments for unruptured brain arteriovenous malformations (AVMs). ${ }^{19}$ On the contrary, a clear shortand midterm benefit ${ }^{4,11,31}$ is evident for ruptured lesions, in order to prevent rerupture. With a reported yearly risk of $6 \%-18 \%,,^{2,411}$ rerupture remains the most important factor for predicting disability and mortality ${ }^{11}$ associated with these lesions. According to Stapf et al., hemorrhagic risk is even higher for ruptured lesions with deep localizations and exclusively deep venous drainage, increasing up to $34.4 \%$ per year. ${ }^{30}$

Although necessary, invasive treatment for AVMs in deep or eloquent locations remains problematic in many cases. Increased complication rates and moderate technical outcomes are described for neurosurgical techniques ${ }^{7,9,10,16,25}$ regarding treatment for basal ganglia or paraventricular AVMs. ${ }^{8}, 30$ Moreover, such lesions often have arterial feeders of very narrow caliber, with very tortuous course or "en passage" arterial supply. Such angio-

ABBREVIATIONS AVM = arteriovenous malformation; DSA = digital subtraction angiography; GCS = Glasgow Coma Scale; $m$ RS = modified Rankin Scale; SRS = stereotactic radiosurgery.

SUBMITTED July 25, 2014. ACCEPTED September 30, 2014.

INCLUDE WHEN CITING Published online March 20, 2015; DOI: 10.3171/2014.9.JNS141714.

DISCLOSURE The authors report no conflict of interest concerning the materials or methods used in this study or the findings specified in this paper. 
architectural features represent technical challenges for endovascular transarterial embolization. Lower total occlusion and higher complication rates have been reported for endovascular treatment of AVMs in eloquent and deep locations. ${ }^{22}$

Stereotactic radiosurgery (SRS) is usually a feasible treatment modality. Nevertheless, the effective radiation dose is attenuated in deep localizations and higher radiation doses are needed to increase effectiveness, which may result in higher complication rates. ${ }^{14}$ The presence of a 2-year mean latency period for cure, ${ }^{23}$ during which the patient is not protected against rerupture, ${ }^{6}$ is also a point to consider in the therapeutic decision-making process.

In many cases withholding treatment remains a reality in everyday clinical practice for several ruptured AVMs, ${ }^{7}$ with challenging angioarchitecture or localizations. Singlesession transvenous endovascular embolization represents an interesting treatment option for several of these cases. In our experience it provides protection from rebleeding and good clinical outcome for patients with otherwise poor clinical perspective due to incurable brain AVMs.

\section{Methods}

This is a prospective, single-center case series extending from January 2008 to June 2013. Therapeutic decisions were made on a multidisciplinary basis. All procedures were performed by the same team of experienced consultant interventional neuroradiologists and supporting personnel. Imaging and clinical evaluation, embolization technique, and follow-up were performed according to preestablished protocols and were entered into a prospectively maintained database. Data were cross-checked by a consultant neuroradiologist.

\section{Clinical Decision Making}

Therapeutic alternatives were discussed among neurosurgeons and neurointerventionalists in a multidisciplinary decision-making process. The series included consecutive patients with ruptured AVMs in the presence of the following criteria (Table 1): 1) high Spetzler-Martin grades; 2) lesions in deep or eloquent locations involving basal ganglia or paraventricular regions, those having choroidal or deep white matter components, or those situated in eloquent cortical regions; 3) unfavorable angioarchitecture, evaluated by digital subtraction angiography (DSA) as the absence of arterial access in cases of residual nidus after previous embolizations, SRS, or neurosurgery, or arterial access that is considered inadequate to obtain complete occlusion by intraarterial embolization due to the following angioarchitectural reasons: very narrow arterial feeder(s), very tortuous course, or en passage arterial feeders; 4) single draining vein; 5) relatively small nidus or nidus remnants that were either inaccessible by a transarterial microcatheter or remained unattained by previous transarterial embolization; and 6) patients referred by other centers after unsuccessful treatment attempts using neurosurgical, endovascular, or radiosurgical techniques.

\section{Clinical Evaluation}

Each patient underwent full clinical and neurological
TABLE 1. Selection criteria for the transvenous AVM embolization technique*

\begin{tabular}{l}
\hline \multicolumn{1}{c}{ Criteria } \\
\hline High Spetzler-Martin grades \\
\hline Nidus location \\
Basal ganglia \\
Paraventricular \\
Choroidal \\
Deep white matter \\
Eloquent cortex \\
\hline Unfavorable angioarchitecture on selective DSA \\
Arterial access to obtain complete occlusion by transarterial embo- \\
$\quad$ lization, due to: \\
$\quad$ Very narrow arterial feeder(s) \\
$\quad$ Very tortuous arterial course \\
$\quad$ En passage arterial feeders \\
\hline Failure of cure w/ classic techniques \\
Neurosurgical \\
Transarterial embolization \\
Radiosurgery \\
\hline Single draining vein \\
\hline Relatively small nidus size or unattained nidus remnant \\
Inaccessible by transarterial microcatheterism \\
Absence of arterial access in cases of residual nidus \\
\hline * Each criterion has additive value to the decision-making process.
\end{tabular}

evaluation by a senior neuroradiologist and a senior anesthesiologist before treatment, at awakening, at discharge, and at 6-month follow-up. Glasgow Coma Scale (GCS) scores were assessed before and after treatment. Modified Rankin Scale (mRS) scores were assessed at the same evaluations.

\section{AVM Classification and Angioarchitecture Study}

Localization was defined by means of MR angiography and DSA findings. Nidus size was measured as the maximum diameter in centimeters for each lesion before transvenous embolization, immediately postembolization, and at follow-up, as well as before any previous treatment was initiated.

Selective and superselective DSA runs were used to identify, study, and register arterial feeders and venous drainage. The following specific angioarchitectural characteristics were recorded: intranidal aneurysm, afferent artery aneurysm, circle of Willis aneurysm, venous stenosis, venous reflux, and venous ectasia. Spetzler-Martin grades ${ }^{28}$ before transvenous embolization and initially (before any treatment) were assessed.

\section{Embolic Agents}

The liquid embolic agent used for transvenous embolization was ethylene vinyl alcohol copolymer (Onyx-18, eV3). Adjunctive loose coiling was considered in cases of large draining veins.

\section{Endovascular Transvenous Embolization Protocol}

Informed consent was obtained from the patient or the patient's relatives in all cases. Procedures were performed 
in a biplane flat panel angiography suite (Allura Xper FD20, Philips Healthcare). Treatment was conducted under general anesthesia with continuous pressure monitoring. Intravenous $3000 \mathrm{IU}$ heparin was administered before selective intracranial vessel navigation.

A 6-F right femoral arterial sheath was placed, and full selective DSA was performed before each treatment. Under road mapping, after catheterization of a supraaortic axis, the right or left jugular vein was punctured, according to the AVM location, and a 6-F sheath was placed. Rotational 3D angiography images with volumetric reconstruction were generated to further evaluate the nidus volume, afferent arteries, and draining veins and to determine the working projections.

A 6-F guiding catheter (Navien, eV3; or ENVOY Codman Neuro, Johnson \& Johnson) was advanced over a 0.035 -in guidewire (Terumo, Europe N.V.) in the proximal part of the venous outlet, which in most cases was the straight sinus. A flexible, dimethyl sulfoxide-compatible microcatheter (1.5/2.7-F Marathon, eV3) was then navigated coaxially to selectively catheterize the AVM draining vein, usually the internal cerebral vein, choroidal veins, or the basal vein (Table 2).

A 5-F vertebral catheter or a 6-F guiding catheter (ENVOY) was positioned arterially, in the cervical internal carotid artery or $\mathrm{V}_{2}-\mathrm{V}_{3}$ segment of the vertebral artery, depending on the AVM location. The catheter was used for control injections, and, in cases of double arterial and venous treatments, a second microcatheter was advanced coaxially as close as necessary to the nidus. In most cases, when ethylene vinyl copolymer (Onyx-18) was the embolic agent of choice for the transarterial injection, a detachable tip microcatheter (Apollo 1.5 and $3 \mathrm{~cm} / 2.7-\mathrm{F}, \mathrm{eV} 3$ ) was used.

All superselective catheterizations were performed under roadmapping. Superselective angiograms were systematically obtained, aiming to evaluate the microarchitecture and hemodynamic behavior of each pedicle. Microcatheter tip position(s), embolic agent injection parameters, and extent of potential embolic agent reflux were chosen accordingly.

Venous or arterial embolic agent reflux during embolization was considered safe only at the segment receiving blood exclusively by the nidus or when the arterial segment was supplying exclusively the nidus, respectively. These were considered safety margins and were respected in all cases. In double transarterial and transvenous embolization cases, injection of the embolic agent would start by the intraarterial microcatheter to reduce nidus inflow, and subsequently transvenous injection would follow, aiming at a retrograde filling of the nidus.

All patients underwent brain CT scanning before and after treatment in the angiography suite according to a dedicated 3D rotational angiography protocol. All patients were hospitalized in the ICU with 24-hour monitoring for at least 48 hours postintervention.

\section{Pre- and Postintervention Imaging Evaluation Angiography}

Preembolization, immediate postembolization, and follow-up angiographic results at 6 and 18 months postembo- lization were classified as total exclusion, small residual nidus ( $<3 \mathrm{~mm}$ in diameter), or residual nidus as a percentage of the total volume of AVM. Four-axis selective DSA was performed at 6 months postintervention, and, if complete occlusion was confirmed, MRI was performed at the 24-month follow-up.

\section{MRI}

All patients systematically underwent brain MRI at 24-28 hours before and after treatment, performed in a 3-T system (Achieva, Philips Medical System), with an imaging protocol of a T2-weighted spin-echo sequence with FLAIR, a T2*-weighted sequence, a 3D T1-weighted sequence, and diffusion-weighted imaging (diffusion gradients in $\mathrm{x}, \mathrm{y}$, and $\mathrm{z}$ directions with $2 \mathrm{~b}$ values $(0$ and 1000 $\mathrm{sec} / \mathrm{mm}^{2}$ ) to evaluate the potential presence of arterial and venous ischemia.

\section{Statistical Analysis}

Descriptive statistical analysis was performed using the Student t-test for quantitative data, after appropriate testing for normal distribution (de Agostino-Pearson test) and the chi-square test for qualitative data. Mean values are presented as the mean $\pm \mathrm{SD}$.

\section{Results}

Among 98 patients with brain AVMs treated between January 2008 and June 2013, 20 patients with ruptured AVMs were selected to be treated by transvenous endovascular embolization.

\section{Patients' Baseline Characteristics}

Ten male and 10 female patients (mean age $37 \pm 18$ years, range 12-64 years) harbored brain AVMs that were considered incurable with classic endovascular and neurosurgical techniques (Fig. 1) and were treated by transvenous embolization over a chronological period of 4.5 years. Among these patients, $12(60.0 \%)$ were treated with a single-session transvenous embolization procedure in the setting of acute or subacute intracranial hemorrhage. In 3 patients (15.0\%) 1 transarterial embolization session preceded the final transvenous embolization session. In 3 patients (15.0\%) 2 transarterial embolization sessions were performed before the final transvenous embolization session, and in 2 patients (10.0\%) 3 transarterial embolization sessions were performed before the final transvenous embolization.

Overall, 8 patients $(40.0 \%)$ had been treated with staged treatments before the final transvenous session. Of these patients, 3 were referred to us by other hospitals after rerupture of partially treated AVMs. In one of these patients (Case 2) 2 previous embolization sessions had been performed. In the second patient (Case 11) multimodality treatment including neurosurgery, 2 embolization sessions, and radiosurgery had been performed before the patient was referred to us. Stereotactic radiosurgery had already been attempted in the third patient (Case 12).

Thirteen patients (65.0\%) experienced an AVM rupture with intracranial hematoma and intraventricular hemorrhage, and the remaining 7 patients (35.0\%) had intracra- 


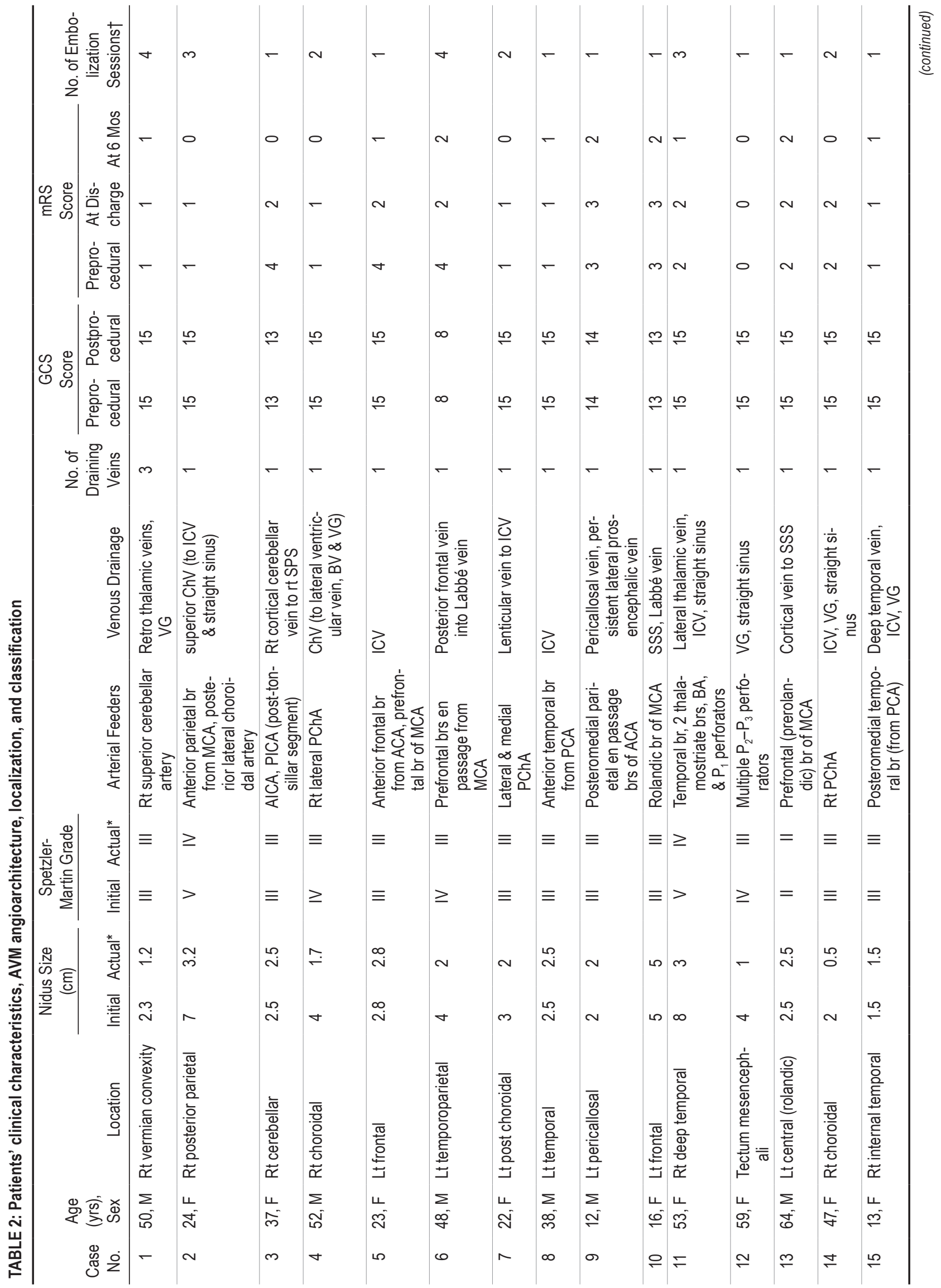




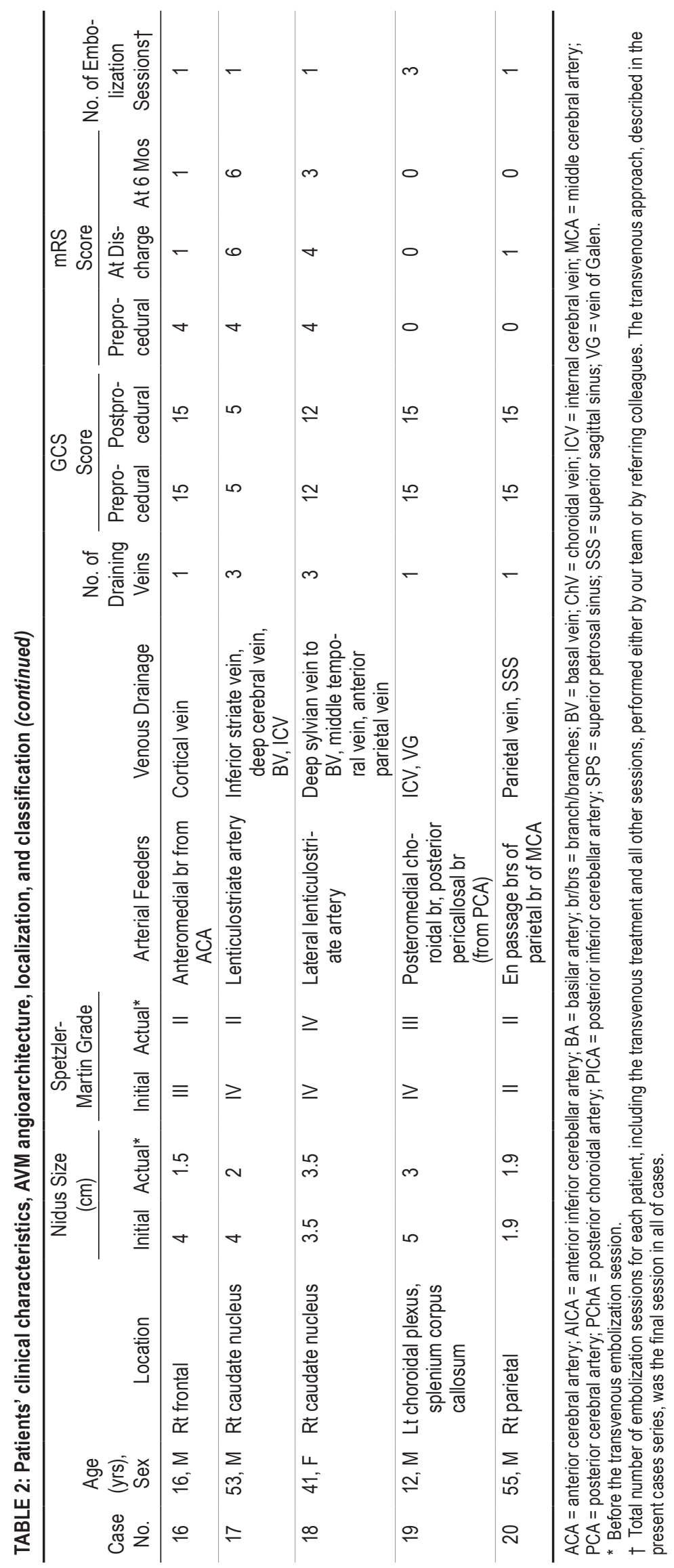



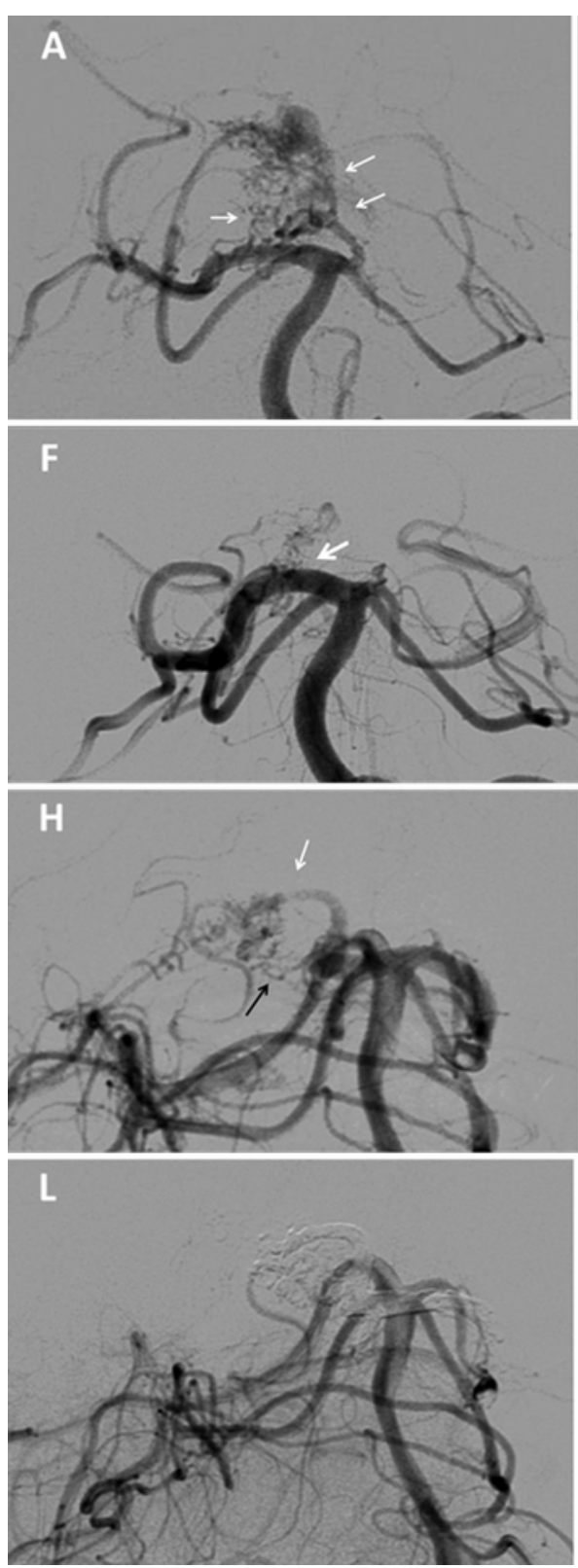
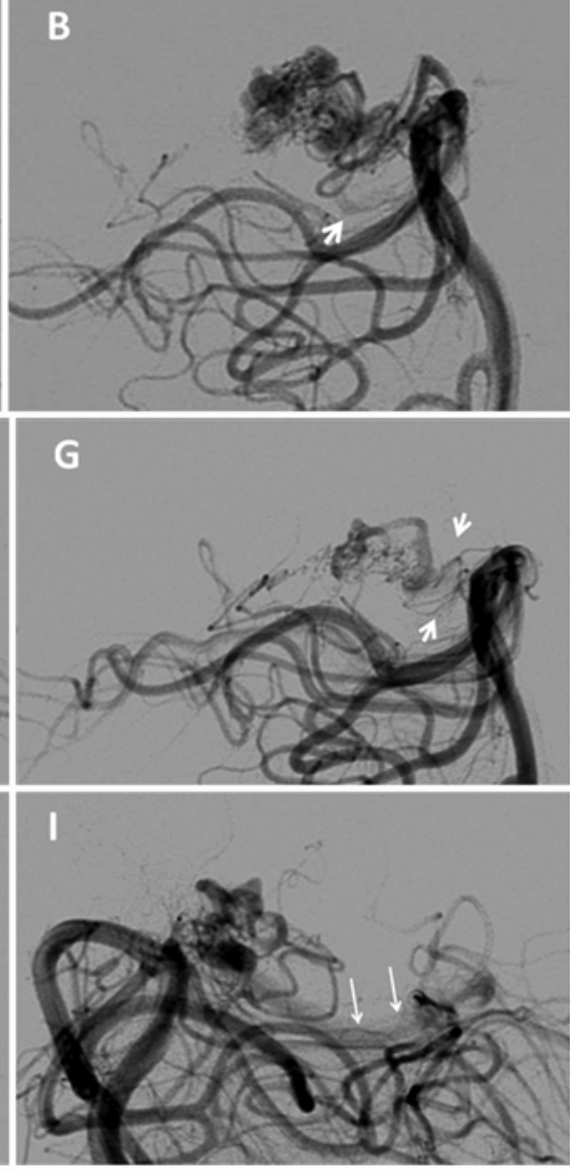

M

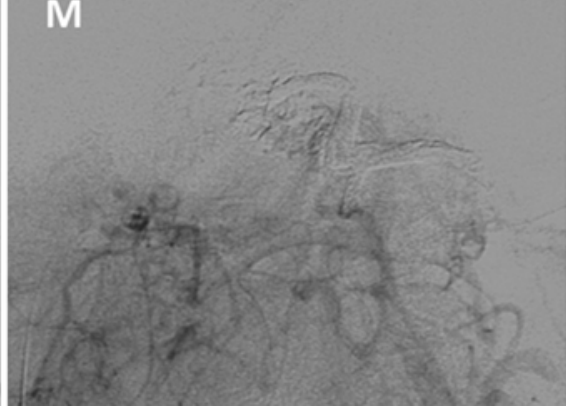

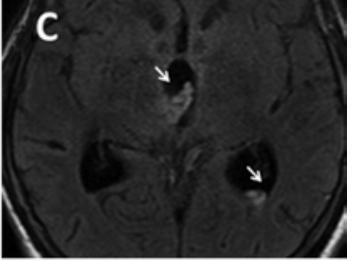
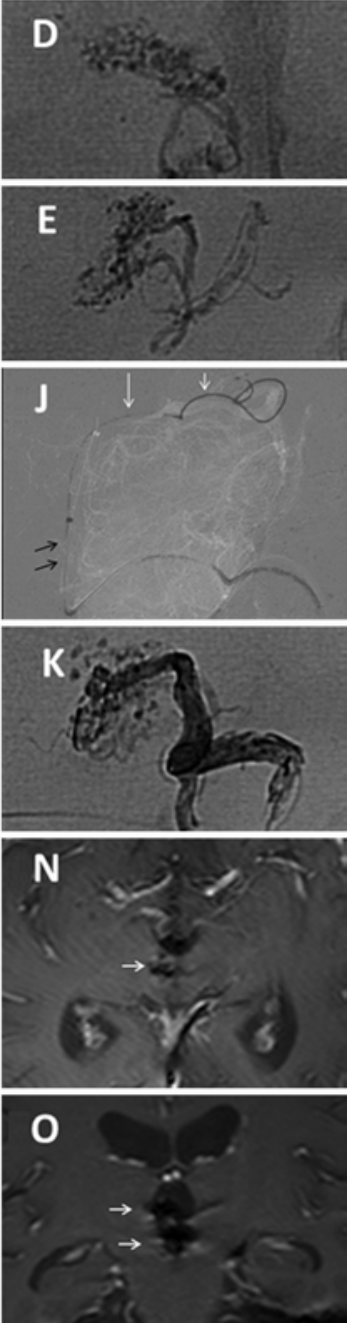

FIG. 1. This 52-year-old man presented to the emergency department with severe, compression-type headaches and meningeal syndrome, due to intraventricular hemorrhage. A and B: Selective right vertebral artery injection DS angiograms (anteroposterior $[A]$ and lateral $[B]$ views). A right choroidal-mesencephalic (tectal plate) Spetzler-Martin Grade IV AVM fed by the lateral posterior choroidal artery ( $\mathrm{A}$, arrows) and draining by single venous outlet via the right choroidal vein to the lateral ventricular vein ( $B$, arrow) was discovered and embolized by superselective intraarterial $N$-butyl cyanoacrylate injection in the acute phase. C: Axial MR image showing the intraventricular hemorrhage (arrows). D and E: Single-shot DS angiograms, at the working projections, showing the N-butyl cyanoacrylate cast. F and G: Selective right vertebral artery injection DS angiograms (anteroposterior [F] and lateral $[\mathrm{G}]$ views) obtained 1 month later. At this time, a second embolization session was performed by transvenous embolization. This session was undertaken with the goal of excluding the residual nidus, which was inaccessible because it was fed by small and tortuous arterial feeders (arrows). $\mathrm{H}$ and I: Selective left VA DS angiograms (oblique views) showing the residual AVM fed by tortuous arterial feeders (black arrow) and drained by the choroidal vein to the basal vein (white arrows). J: Road map, lateral view. Access was via the vein of Galen, basilar vein, and lateral ventricular vein. The microcatheter is positioned in the right choroidal vein. The guiding catheter is navigated in the straight sinus (black arrows), and the microcatheter is navigated in the lateral ventricular and choroidal veins (white arrows). K: Single-shot DS angiogram showing the cast of Onyx-18. L and M: Oblique views obtained immediately postoperatively showing complete exclusion of the AVM. At the 6-month angiographic follow-up persistence of total occlusion was confirmed (not shown). $\mathrm{N}$ and $\mathrm{O}$ : Coronal $(\mathrm{N})$ and sagittal $(\mathrm{O}) \mathrm{T1}$-weighted Gd-administered MR images showing the cast of Onyx in the nidus and paraventricular involvement. The patient's clinical situation was unchanged after embolization. The patient's mRS score at discharge was 1, and at the 6-month follow-up the mRS score was 0. 
nial hematoma without intraventricular involvement. In one patient (Case 18; 5.0\%) the hematoma was resected before embolization, and in another patient (5.0\%) surgery for hematoma resection was performed after embolization (Case 17). A ventriculostomy was placed before embolization in 4 patients $(20.5 \%)$ and after embolization in 1 patient $(5.0 \%)$. In all cases in which surgery was performed after transvenous embolization, the intent was to drain the preexisting hematoma and not because hematoma volume had increased postembolization.

The initial Spetzler-Martin grade, before we or other clinicians and specialists initiated any treatment, was II for 2 patients $(10.0 \%)$, III for 10 patients $(50.0 \%)$, IV for 6 patients $(30.0 \%)$, and $\mathrm{V}$ for 2 patients $(10.0 \%)$. Before the transvenous embolization session, the Spetzler-Martin grades were II for 4 patients (20.0\%), III for 13 patients $(65.0 \%)$, and IV for 3 patients $(15.0 \%)$. The GCS scores before treatment were 15 for 14 patients (70.0\%); 14 for 1 patient (5.0\%); 13 for 2 patients (10.0\%); and 12,8 , and 5 for 1 patient each $(5.0 \%)$. The mRS scores before treatment were 0 for 3 patients $(15.0 \%), 1$ for 6 patients $(30.0 \%), 2$ for 3 patients (15.0\%), 3 for 2 patients $(10.0 \%)$, and 4 for 6 patients $(30.0 \%)$.

\section{Localization, Angioarchitecture, and AVM Characteristics}

Seventy-five percent (15 cases) of the AVMs were supratentorial with at least a deep component, among which $25.0 \%$ (5 AVMs) involved the choroidal plexuses, $35.0 \%$ (7 AVMs) involved deep white matter or basal ganglia, and $15.0 \%$ (3 AVMs) were exclusively deep in location, while $15.0 \%$ (3 AVMs) were supratentorial (exclusively cortical) and $10.0 \%$ (2 AVMs) were infratentorial.

Venous drainage was exclusively deep in 14 cases (70.0\%), involving the deep venous system in 2 cases $(10.0 \%)$ and exclusively cortical in 4 cases $(20.0 \%)$. In the vast majority of cases (17 [85.0\%]) the drainage involved a single venous outlet. In the remaining 3 cases $(15.0 \%)$ drainage involved 3 venous outlets. The exact locations, arterial supply, and venous outlets for these AVMs are summarized in Table 2 .

The mean nidus size before transvenous embolization was $2.3 \pm 1.1 \mathrm{~cm}$; thus, most nidi were relatively small. The mean initial nidus size of the AVMs before any previous therapeutic approach was $3.7 \pm 1.7 \mathrm{~cm}$. Specific angioarchitectural characteristics retained were intranidal aneurysm in 3 cases (15.0\%; Cases 7, 10, and 17), afferent artery aneurysm in 1 case $(5.0 \%$; Case 11$)$, venous stenosis in 2 cases (10.0\%; Cases 2 and 9), and localized venous ectasia in 1 case $(5.0 \%$; Case 6$)$.

\section{Procedure/Technique Specifications}

Technical feasibility was $100 \%$. The mean Onyx-18 volume used was $1.2 \pm 1.7 \mathrm{ml}$ (range $0.4-2.4 \mathrm{ml}$ ). All procedures were performed with transvenous embolization; in 11 of 20 cases $(55.0 \%)$ arterial pedicles were also embolized (in 1 case a double arterial catheterization with Onyx-18 injection was performed). In 3 cases (15.0\%) loose coiling of the vein was undertaken via the venous approach to reduce venous outflow and to obtain a sufficient retrograde propagation of the liquid embolic agent. The decision to use this modified technique was based on the presence of high-flow and large-caliber venous outlet, and partial/loose coiling was undertaken, with the goal of slowing down the flow rather than obstructing the vein.

Embolization access involved the straight sinus in 13 cases (65\%) (the internal cerebral vein in 6 [30\%; Fig. 2], the basal vein in 3 [15\%], choroidal veins in 3 [15\%], and a retro-thalamic vein in 1 [5\%]), cortical veins via the superior sagittal sinus in 3 cases $(15 \%)$, venous tributaries to the vein of Labbé in 2 cases $(10 \%)$, the cerebellar cortical vein through the superior petrosal sinus in 1 case $(5 \%)$, and a persistent lateral prosencephalic vein in 1 case $(5 \%)$.

\section{Technical Difficulties and Procedure-Related Complications}

One $(5.0 \%)$ intraprocedural venous rupture occurred (Case 13). Nevertheless, the procedure was continued until complete occlusion of the nidus was achieved, without clinically evident consequences. One arterial pedicle dissection (5.0\%; Case 5) that occurred during the procedure was also managed without clinical consequence. In this case the transvenous injection allowed for a complete eradication of the nidus.

One procedure-related complication (Case 20) occurred during postoperative hospitalization in a patient with a cortical and white matter right parietal AVM with single cortical venous drainage to the superior sagittal sinus, embolized in one session by a single transvenous approach. Within 24 hours of embolization, the patient developed MRI-confirmed hemorrhagic transformation of a venous infarction surrounding the nidus. At discharge the patient had an mRS score of 1 .

\section{Events Unrelated to the Embolization}

One postprocedural death, unrelated to the embolization procedure, occurred during the hospitalization period in a patient with a right caudate nucleus AVM (Case 17) who presented with tetraventricular hemorrhage, dilation of the third and fourth ventricles, and a caudate nucleus hematoma. The patient's GCS score at presentation was 5. An uncomplicated transvenous embolization was performed before surgery, with total exclusion of the nidus. Routine postoperative CT scanning in the angiography suite showed absence of rebleeding. The patient's condition deteriorated following hematoma resection, which was performed in a further attempt to alleviate intracranial pressure since external ventriculostomy was not sufficient.

\section{Clinical, Angiographic, and Imaging Outcomes and Follow-Up}

Complete exclusion was achieved in 19 cases (95\%); a small residual nidus of less than $2 \mathrm{~mm}$ was left in 1 case (5\%; Case 2). This patient was referred for SRS after embolization. For all other patients, complete exclusion of the nidus was confirmed on angiograms obtained at the 6-month and 18-month follow-up visits.

All patients $(100 \%)$ were clinically stable after embolization. Patients' postembolization GCS scores were unchanged from preembolization status. The postembolization clinical situation was unchanged for all patients.

At discharge the mRS scores were as follows: 0 for 2 


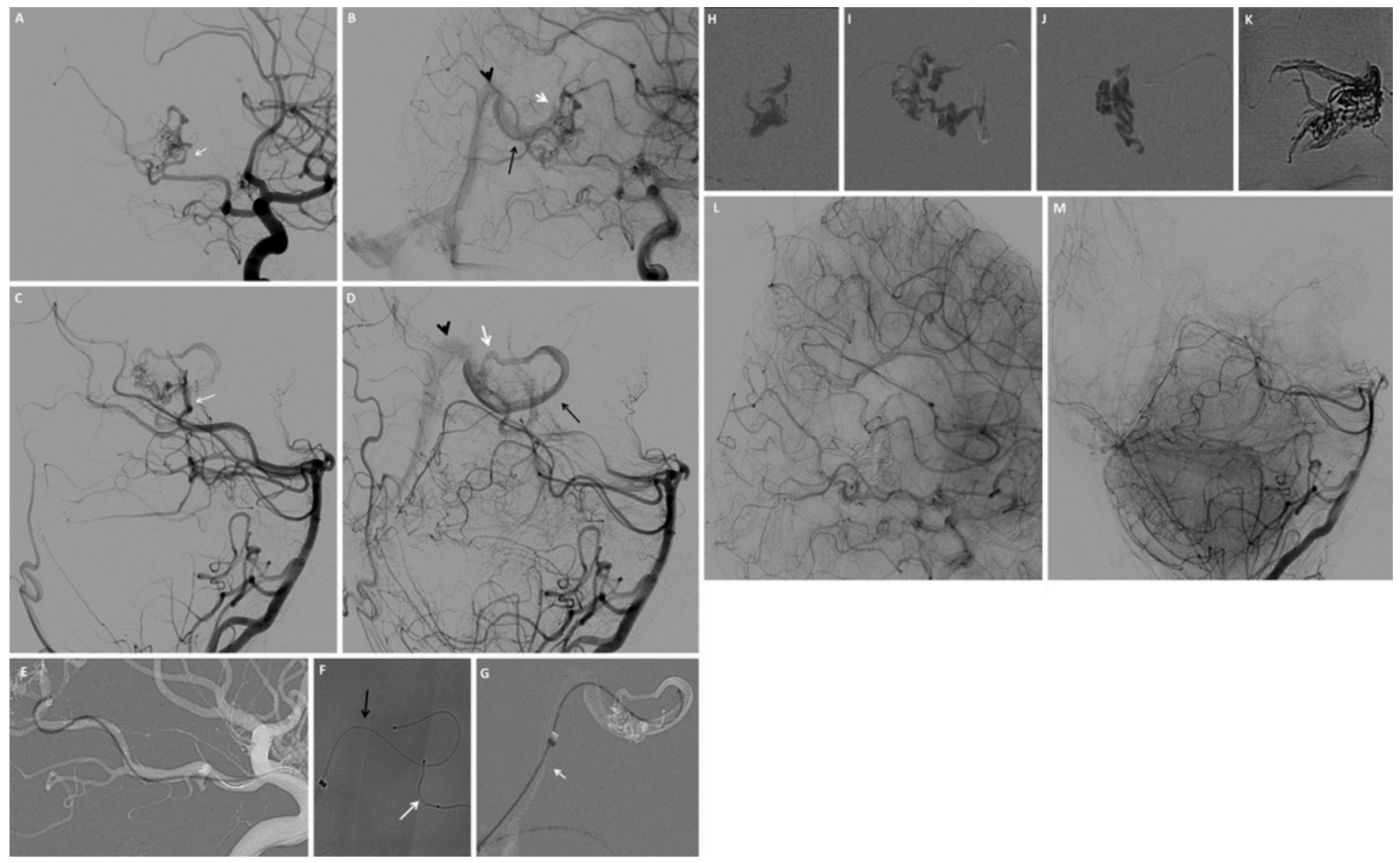

FIG. 2. This 13-year-old girl harbored a Spetzler-Martin Grade III left medial temporal AVM revealed by left posteromedial temporal and cingular hematoma. The patient's GCS score before embolization was 15. A-D: Selective left internal carotid artery injection (oblique $[A]$ and lateral $[B]$ views) and selective left vertebral artery injection (anteroposterior [C] and lateral [D] views) DS angiograms showing a 1.5-cm AVM nidus, fed by a feeder depending on the left posterior temporal branch of the left posterior cerebral artery (thin white arrows in A and C) and drained by a single venous outlet (thick white arrows in B and D), into the internal cerebral vein (black arrow) and the vein of Galen (arrowheads). E: Angiographic roadmap showing superselective microcatheterization with a $1.5-\mathrm{cm}$ detachable-tip microcatheter. G: Roadmap showing Superselective venous microcatheterization via a 6-F guiding catheter left in the straight sinus (arrow). F: Arterial (white arrow) and venous (black arrow) microcatheters are in place for embolization. H: Single-shot DS angiogram showing transarterial embolic agent (Onyx-18) injection. I and J: Single-shot DS angiograms progressive transvenous (Onyx-18) injection. K: Single-shot DS angiogram showing the cast of embolic material immediately postoperatively. $L$ and M: Selective left internal carotid $(L)$ and vertebral artery $(M)$ injection DS angiograms showing complete nidus exclusion. The patient's clinical situation was unchanged postembolization. Her mRS at 6 months was 1. Angiography performed at 6 months demonstrated complete exclusion of the nidus.

patients (10.0\%), 1 for 8 patients $(40.0 \%), 2$ for 6 patients $(30.0 \%), 3$ for 2 patients $(10.0 \%)$, and 4 and 6 for 1 patient each (5.0\%). At 6 months postembolization the mRS scores were as follows: 0 for 8 patients $(40.0 \%), 1$ for 6 patients (30.0\%), 2 for 4 patients $(20.0 \%), 3$ for 1 patient (5.0\%), and 6 for 1 patient $(5.0 \%)$ The clinical condition of the patient with an mRS score of 6 deteriorated after hematoma resection. This represents the overall mortality of the series (5\%). Procedure-related mortality was $0 \%$. Overall, at 6-month follow-up $90 \%(\mathrm{n}=18)$ of the patients were independent in their everyday lives.

\section{Discussion}

Deep-seated brain AVMs represent a real challenge for all invasive therapeutic approaches. In neurosurgery, high Spetzler-Martin ${ }^{28,29}$ grades ( $\geq$ III) and deep brain locations $^{24}$ are associated with high complication rates. ${ }^{22} \mathrm{Sev}$ eral surgical technical modifications, ${ }^{18}$ such as excision of "non-eloquent" brain parts to obtain surgical access, ${ }^{12}$ as well as multimodality treatments, ${ }^{16}$ have been described in small case series as solutions for deep AVM treatment with moderate clinical results.

Few articles describe transarterial embolization for the treatment of deep or inaccessible brain AVMs; these papers consist of relatively small case series. ${ }^{21,23}$ The authors of these studies reported total occlusion rates varying between $2.6 \%$ and $43 \%$, often with SRS as an adjunctive modality for residual nidus treatment. The main technical impediment encountered was narrowness and tortuosity of the arterial pedicle. In a multivariate analysis of predictive factors for complications in AVM embolization, ${ }^{17}$ Spetzler-Martin Grades III-V were independent factors of poor outcome after embolization.

To the best of our knowledge, apart from very few case reports, ${ }^{1,15}$ this is the first case series of brain AVM treatment via a transvenous endovascular approach. Although in the past transvenous endovascular techniques were con- 
sidered risky, ${ }^{5,13}$ the concept of venous side occlusion for permanent AVM cure has been around for 2 decades. ${ }^{20}$ Advances in liquid embolic materials have allowed for development and evolution of this technique. We have been using the presented protocol during the past few years in select cases,,$^{15}$ and, in our experience, when rules are respected there is no increased hemorrhagic risk over intraarterial techniques. However, it is important to maintain an appropriate injection rate to exclude the nidus before occluding the initial part of the main draining venous outlet.

We present a case series of patients with high initial Spetzler-Martin grades $(90 \%$ of the cases had SpetzlerMartin grades $\geq$ III) and AVMs in highly eloquent locations, with several factors predisposing them to rebleeding. Most of these AVMs were in deep locations, with deep, single venous drainage patterns; in $25.0 \%$ of cases there were intraventricular components. Some of the lesions had undergone previous multimodality treatments, including SRS, without obtaining total exclusion.

The lesions were fed by very narrow and tortuous arterial pedicles that were not suitable for navigation or for curative intraarterial injection. A transvenous approach was the only alternative to withholding treatment in these cases (Fig. 1). Nevertheless, transvenous access was generally attained with relative ease. The only technical complication regarding venous navigation occurred in a cortical vein. Our observation overall is that deep veins seem much more suitable for navigation than cortical ones.

Although invasive treatments of highly eloquent lesions are associated with poor clinical outcome, ${ }^{27}$ in our series $90 \%$ of the patients had mRS scores of 0-2 at 6 months. Moreover, our rate of single-session total nidus obliteration $(95 \%)$ is the highest reported so far, even compared with series with cortical localizations or lower SpetzlerMartin grades. ${ }^{19,26}$ This fact accounts for the good clinical outcome of the patients, since rerupture did not occur in the postprocedural period.

Our results are not in accordance with recent prospective randomized data regarding treatment of unruptured brain AVMs,${ }^{19}$ even though our series represents a much riskier population in terms of technical and overall complications. It is evident that data are not comparable due to study design and population type (ruptured AVMs) and small sample size; still, with an $85.0 \%$ percentage of $\geq$ III Spetzler-Martin grade lesions, a 0\% rerupture rate, and a $5 \%$ procedure-related complication rate the technique seems safe and effective.

We attribute these results to the following: 1) The treatment strategy used is adapted to the specific angioarchitectural characteristics of the population, allowing otherwise inaccessible AVMs to be treated without compromising safety. 2) The strategy allows for a high total nidus obliteration rate. 3) The absence of a latency period for cure protects the patients from being exposed to an increased bleeding risk.

We only encountered 1 procedure-related complication, an MRI-confirmed postoperative hemorrhagic transformation of venous ischemia, attributed to venous hyperemic complication $^{3}$ in a patient with total nidus occlusion. In the setting of cortical single venous outlet, the draining vein was joined at its distal part by a cortical vein draining normal cerebral parenchyma. After embolization, the venous outlet was not obstructed at the level of the convergence, but acute pressure rearrangement inside the veins resulted in venous congestion, with subsequent compromise of the blood-brain barrier. Our observation is in accordance of a recent study on venous flow patterns regarding treatment risks of brain AVMs. ${ }^{3}$

\section{Limitations}

Even though our case series has a prospective design, the study is subject to limitations due to the small number of cases. Larger studies are needed to confirm our experience. Despite this fact, we feel that the technique presented represents an interesting alternative treatment in similar complex AVM cases. The technique seems to yield very good results after careful case study and selection and is suitable for relatively small AVMs without sufficient arterial access and preferably when deep venous system navigation is used. It can be applied as single-session technique for relatively small AVMs or as final-session technique to ensure complete nidus eradication in otherwise incurable AVMs.

\section{Conclusions}

Transvenous Onyx-18 embolization appears to be a safe and effective single-session alternative to withholding treatment or performing time-consuming staged treatments in cases of complex brain AVMs with high Spetzler-Martin grades, especially for deep veins and relatively small nidus sizes. It may be used as last-session treatment in larger AVMs, for parts of the lesions that remain otherwise inaccessible, to ensure complete nidal occlusion.

\section{References}

1. Consoli A, Renieri L, Nappini S, Limbucci N, Mangiafico S: Endovascular treatment of deep hemorrhagic brain arteriovenous malformations with transvenous onyx embolization. AJNR Am J Neuroradiol 34:1805-1811, 2013

2. Crawford PM, West CR, Chadwick DW, Shaw MD: Arteriovenous malformations of the brain: natural history in unoperated patients. J Neurol Neurosurg Psychiatry 49:1-10, 1986

3. D’Aliberti G, Talamonti G, Piparo M, Debernardi A, Zella S, Boccardi E, et al: Venous flow rearrangement after treatment of cerebral arteriovenous malformations: a novel approach to evaluate the risks of treatment. World Neurosurg 82:160169,2014

4. da Costa L, Wallace MC, Ter Brugge KG, O'Kelly C, Willinsky RA, Tymianski M: The natural history and predictive features of hemorrhage from brain arteriovenous malformations. Stroke 40:100-105, 2009

5. Dandy WE: Contributions to brain surgery: A. removal of certain deep-seated brain tumors B. Intracranial approach with concealed incisions. Ann Surg 82:513-525, 1925

6. Fabrikant JI, Levy RP, Steinberg GK, Phillips MH, Frankel KA, Lyman JT, et al: Charged-particle radiosurgery for intracranial vascular malformations. Neurosurg Clin N Am 3:99-139, 1992

7. Ferch RD, Morgan MK: High-grade arteriovenous malformations and their management. J Clin Neurosci 9:37-40, 2002

8. Fleetwood IG, Marcellus ML, Levy RP, Marks MP, Steinberg GK: Deep arteriovenous malformations of the basal ganglia and thalamus: natural history. J Neurosurg 98:747-750, 2003 
9. Hamilton MG, Spetzler RF: The prospective application of a grading system for arteriovenous malformations. Neurosurgery 34:2-7, 1994

10. Hernesniemi J, Romani R, Lehecka M, Isarakul P, Dashti R, Celik O, et al: Present state of microneurosurgery of cerebral arteriovenous malformations. Acta Neurochir Suppl 107:71-76, 2010

11. Hernesniemi JA, Dashti R, Juvela S, Väärt K, Niemelä M, Laakso A: Natural history of brain arteriovenous malformations: a long-term follow-up study of risk of hemorrhage in 238 patients. Neurosurgery 63:823-831, 2008

12. Heros RC: Brain resection for exposure of deep extracerebral and paraventricular lesions. Surg Neurol 34:188-195, 1990

13. Houdart E, Gobin YP, Casasco A, Aymard A, Herbreteau D, Merland JJ: A proposed angiographic classification of intracranial arteriovenous fistulae and malformations. Neuroradiology 35:381-385, 1993

14. Karlsson B, Lax I, Söderman M: Factors influencing the risk for complications following Gamma Knife radiosurgery of cerebral arteriovenous malformations. Radiother Oncol 43:275-280, 1997

15. Kessler I, Riva R, Ruggiero M, Manisor M, Al-Khawaldeh M, Mounayer C: Successful transvenous embolization of brain arteriovenous malformations using Onyx in five consecutive patients. Neurosurgery 69:184-193, 2011

16. Lawton MT, Hamilton MG, Spetzler RF: Multimodality treatment of deep arteriovenous malformations: thalamus, basal ganglia, and brain stem. Neurosurgery 37:29-36, 1995

17. Ledezma CJ, Hoh BL, Carter BS, Pryor JC, Putman CM, Ogilvy CS: Complications of cerebral arteriovenous malformation embolization: multivariate analysis of predictive factors. Neurosurgery 58:602-611, 2006

18. Liu KD, Lee LS: Microsurgical treatment of deep arteriovenous malformations-basal ganglia and thalamus. Zhonghua Yi Xue Za Zhi (Taipei) 64:23-30, 2001

19. Mohr JP, Parides MK, Stapf C, Moquete E, Moy CS, Overbey $\mathrm{JR}$, et al: Medical management with or without interventional therapy for unruptured brain arteriovenous malformations (ARUBA): a multicentre, non-blinded, randomised trial. Lancet 383:614-621, 2014

20. Mullan S: Reflections upon the nature and management of intracranial and intraspinal vascular malformations and fistulae. J Neurosurg 80:606-616, 1994

21. Oran I, Parildar M, Derbent A: Ventricular/paraventricular small arteriovenous malformations: role of embolisation with cyanoacrylate. Neuroradiology 47:287-294, 2005

22. Pan J, He H, Feng L, Viñuela F, Wu Z, Zhan R: Angioarchitectural characteristics associated with complications of embolization in supratentorial brain arteriovenous malformation. AJNR Am J Neuroradiol 35:354-359, 2014

23. Paulsen RD, Steinberg GK, Norbash AM, Marcellus ML, Marks MP: Embolization of basal ganglia and thalamic arteriovenous malformations. Neurosurgery 44:991-997, 1999

24. Potts MB, Jahangiri A, Jen M, Sneed PK, McDermott MW, Gupta N, et al: Deep arteriovenous malformations in the basal ganglia, thalamus, and insula: multimodality management, patient selection, and results. World Neurosurg 82:386-394, 2014

25. Potts MB, Young WL, Lawton MT: Deep arteriovenous malformations in the basal ganglia, thalamus, and insula: microsurgical management, techniques, and results. Neurosurgery 73:417-429, 2013

26. Saatci I, Geyik S, Yavuz K, Cekirge HS: Endovascular treatment of brain arteriovenous malformations with prolonged intranidal Onyx injection technique: long-term results in 350 consecutive patients with completed endovascular treatment course. J Neurosurg 115:78-88, 2011

27. Schaller C, Schramm J, Haun D: Significance of factors contributing to surgical complications and to late outcome after elective surgery of cerebral arteriovenous malformations. J Neurol Neurosurg Psychiatry 65:547-554, 1998

28. Spetzler RF, Martin NA: A proposed grading system for arteriovenous malformations. J Neurosurg 65:476-483, 1986

29. Spetzler RF, Martin NA: A proposed grading system for arteriovenous malformations. 1986. J Neurosurg 108:186-193, 2008

30. Stapf C, Mast H, Sciacca RR, Choi JH, Khaw AV, Connolly ES, et al: Predictors of hemorrhage in patients with untreated brain arteriovenous malformation. Neurology 66:1350-1355, 2006

31. Wilkins RH: Natural history of intracranial vascular malformations: a review. Neurosurgery 16:421-430, 1985

\section{Author Contributions}

Conception and design: Mounayer. Acquisition of data: Iosif. Analysis and interpretation of data: Iosif, Saleme, Mounayer. Drafting the article: Iosif. Critically revising the article: Mendes, Saleme, Ponomarjova, Silveira, Caire, Mounayer. Study supervision: Mounayer.

\section{Correspondence}

Christina Iosif, 2 Avenue Martin Luther King, 87042 Limoges, France. email: christina.iosif@gmail.com. 